\title{
Uplink Medium Access Control for WLANs with Multi-beam Access Point
}

\author{
Jianfeng Wang, Yuguang Fang, Dapeng Wu \\ Department of Electrical \& Computer Engineering \\ University of Florida, Gainesville, Florida 32611-6130 \\ Tel: (352) 846-3043, Fax: (352) 392-0044 \\ E-mail: jfwang@ufl.edu, fang@ece.ufl.edu,wu@ece.ufl.edu
}

\begin{abstract}
We consider the CSMA/CA based uplink Medium Access Control (MAC) protocol design for a wireless local area network (WLAN) with the use of multi-beam directional antennas at the access point. Our MAC protocol intends to fully utilize the spatial reuse by allowing as many parallel uplink data transmissions as possible. Since all nodes including the access point run in the contention-based MAC protocol, it is not easy to realize multiple collision-free parallel data transmissions while preserving the ad hoc nature of CSMA/CA based MAC. In addition to improving channel efficiency, we also consider the backward compatibility in our design, whereby a 802.11 limited node can transparently access a multi-beam access point. Our simulation results show that our scheme can improve throughput significantly.
\end{abstract}

Index Terms-Medium Access Control (MAC), Wireless LAN, Directional Antennas

\section{INTRODUCTION}

Considering the popularity of wireless local access, it is of great interests to improve throughput and energy efficiency for data transmission between terminals and an access point. Smart antennas bring two major benefits, spatial reuse and antenna gain, thus promising to increase the throughput and energy efficiency.

In this paper, we investigate the application of multi-beam smart antennas at the access point, which is generally more powerful and of less size-constraints than mobile-terminals (suppose mobile-terminals still use omnidirectional antennas), thus feasible to equip multiple smart antennas. Two types of multi-beam smart antennas are widely discussed in the literature; one is based on fixed-beam directional antennas, another is based on adaptive arrays. Although adaptive-array based smart antennas may work better in multipath rich environments, it poses great complexity on the transceiver and MAC protocol. In this paper, we consider sectorized directional antennas due to its simplicity, availability and attractive cost. Rather than the single-beam directional antenna system, in which only one beam can be utilized at a time, we assume each directional antenna-beam is equipped with one separate radio transceiver. Thus it is possible to utilize the multiple beams simultaneously for transmission or receiving, dramatically increasing the throughput.

This work was supported in part by the U.S. Office of Naval Research under Young Investigator Award N000140210464 and under grant N000140210554.
Although sectorized directional antennas with multiple transceivers have been investigated in the cellular networks for several years, there is not much work for the ad hoc based wireless LANs, in which all nodes, including the access-point, access medium via contention. Due to the well-known cocite inference problem, we need carefully design on MAC to achieve spatial reuse. When all the transceivers run in the same frequency band, the co-site interference forbids transmission in one sectorized beam while receiving in another.

Note the most widely investigated contention-based MAC protocols, including IEEE 802.11 MAC protocol, are based on CSMA. To increase link reliability, 2-way handshake (DATA+ACK, pure CSMA) is used for short packet transmission and 4-way handshake (RTS+CTS+DATA+ACK, CSMA/CA) is used for long packet transmission. Our proposed protocol also follows the CSMA based approach. Contention based medium access, multiple-way handshake, omnidirectional transmission/receiving of mobile-terminals, together with the co-site interference problem at the access point, impose great challenges on the full utilization of the sectorized beams.

The primary goal of our MAC protocol is to exploit multibeam directional antennas for increasing throughput, given the above challenges. Our scheme also considers the backward compatibility with 802.11 , whereby a 802.11 -limited node can access a multi-beam access-point transparently. It is worthy to note that, since all the nodes including the access point run in CSMA/CA based MAC, our proposed MAC protocol is not limited to one-hop; in other words, the uplink medium access control protocol provided in this paper can work well no matter whether the network is single-hop or multihop.

The rest of paper is organized as follows. We first present related works in Section II. Then we detail our proposed protocol in Section III. Simulation results are provided in Section IV. Finally, we conclude the paper in Section V.

\section{RELATED WORKS}

In this paper, we are interested in the contention-based medium access control for multi-beam antennas system. The basic goal of medium access control for multi-beam antennas system is to facilitate multiple transmissions or receptions [2] [3] [4] [5] [6] [7] [8]; intention for extending range is also found in the literature [8]. According to antennas model 
explained in the last section, MAC for multi-beam antennas system can be categorized into two groups, adaptive-array multi-beam antenna system [3]- [8] and fixed multi-beam antennas system [2]. The major difference between adaptivearray multi-beam MAC and fixed multi-beam MAC is that the former need use training process to let beamforming take place before packet transmission, which may cause a lot of overhead.

Our scheme is based on fixed multi-beam antennas system. The major benefit of fixed multi-beam antennas system is its simplicity. Fixed multi-beam antennas system may not work well in multipath rich environments, for example, some indoor environments. However it may be a better choice in comparison with adaptive-array multi-beam system if the line-of-sight is dominant. [2] discusses the application of multiple fixed beam directional antennas in heterogenous ad hoc networks. Co-cite problem (multi-beam node cannot transmit in one beam while receiving in another beam) and receiver blocking problem have been discussed in that paper. Our paper solves the co-cite problem and exploits spatial reuse to the most extent by allowing a multi-beam access point as many parallel data communications as possible; receiver blocking problem is resolved by broadcasting network-allocation-vector(NAV, this field is included in the RTR (ready-to-receive) message sent by the access point) in all beam-sections. In addition, our protocol supports the backward compatibility by allowing 802.11-limited nodes to access a multi-beam access-point transparently.

\section{PROPOSED MAC PROTOCOL}

\section{A. Framework}

Before presenting our scheme, we briefly review the widely used IEEE 802.11 MAC protocol [1]. With DCF (DistributedCoordination-Function) mode 802.11 MAC, each user distributedly contends for channel; after successful exchange of RTS/CTS, DATA of the winning user will be immediately sent, then the ACK. We may easily find that no spatial reuse (provided by sectorized directional antennas) can be achieved if we strictly follow the handshake rule of 802.11 MAC. To facilitate the concurrent use of multiple antenna beams, we need a more coordinated way, while still keeping the ad hoc nature.

Our proposed uplink MAC structure is shown in Fig. 1. All nodes having uplink packets in queue will not contend for channel until the access point sends a ready-to-receive(RTR) message. In other words, the uplink medium access is essentially access-point-initiated. After receiving the RTR message, intended nodes will contend for channel within the contention period $T_{1}$ shown in the Fig. 1. The contention resolution scheme we design will facilitate multiple nodes to win out and the winning nodes will be collision-free with each other. Here the collision-free means the winning nodes will not collide with each other when they send DATA to the access point or receive ACK from the access point in the period of $T_{2}$ and $T_{3}$, respectively. In order to be collision-free, the winning nodes must be in different beam-sections. In the phase of DATA transmission, multiple back-to-back packets are allowed

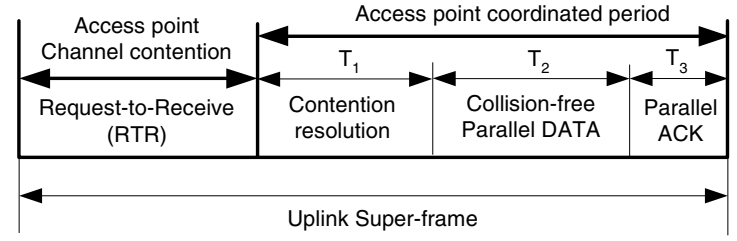

Fig. 1. Uplink superframe

to transmit by one node as long as the total time spent does not exceed the length of $T_{2}$. Note power control and/or auto rate scheme may be incorporated in our scheme to further increase throughput and energy efficiency. In the period of parallel ACK, the access point will transmit one ACK for each user, but concurrently, simply because the users are in different beam-sections.

The access point initiates the uplink medium access in a frequency adaptive to the current traffic load. Since the access point also runs in the ad hoc mode, the access point need to contend for channel as the normal nodes ${ }^{1}$. However, the access point can send RTR messages in a higher priority, i.e., with smaller Inter-frame-space(IFS) and contention window size, than mobile-terminals to exchange RTS messages. We assume the access point still follows the de-facto Exponential-Backoff algorithm to resolve collision with other normal nodes. Thanks to the smaller Inter-frame-space(IFS) and contention window size, the access point will be much easier to capture the channel than mobile terminals. This priority differentiation guarantees that the access point gains higher priority to access medium, which is generally desired.

\section{B. Channel contention}

In this section, we discuss the CSMA based channel contention during period $T_{1}$. The CSMA based RTS/CTS exchange is illustrated in Fig. 2. $T_{i d}, T_{c o l}$, and $T_{\text {suc }}$ are defined as

$$
\left\{\begin{array}{l}
T_{i d}=\sigma \\
T_{c o l}=R T S+D I F S \\
T_{s u c}=R T S+S I F S+C T S+S I F S,
\end{array}\right.
$$

where $\sigma$ is a minislot. Different from traditional CSMA with omnidirectional-antenna access point, two nodes simultaneously transmitting RTS to the access-point will not collide with each other if they are in different beam sections in our proposed system. In other words, if there is only one node sending RTS in a given beam section, it succeeds no matter how many nodes concurrently sending RTSs in other beamsections. Except that all the RTSs collide, the access point will send CTSs in all beam directions concurrently after receiving RTSs. For the beam section in which there is only one RTS, the access point will reply a positive CTS to confirm further data transmission in period $T_{2}$ for the node sending RTS; all the other intended transmission nodes in that beam-section will then keep silent until the end of current access-pointcoordinated-period. For the beam-section in which there are

\footnotetext{
${ }^{1}$ The access point will keep silent in all beam-sections if it senses the channel is busy.
} 


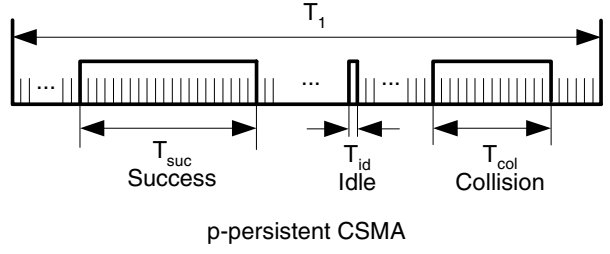

Fig. 2. CSMA based contention resolution scheme for baseline MAC

more than one RTS, the access point will reply a negative CTS to indicate that the contention fails; all the intended transmission nodes including those failed in that beam-section will continue to contend for channel until one node wins out or the period $T_{1}$ ends. For the beam-section in which there is no RTS, the access point will send CTS either, to silent intended transmission nodes in that beam-section for a while, thus reducing the hidden terminal problem (to those in other beam sections who successfully sent RTS and need to receive CTS from the access point); intended transmission nodes in the beam-section that no RTS was sent will continue to contend for channel after receiving CTS, until one node wins out or the period $T_{1}$ ends. If all the received $\operatorname{RTS}(\mathrm{s})$ in all beamsections are collided, the access point may just keep silent. All intended transmission nodes including those who sent the RTS will resume contention after the channel gets to be idle for DIFS.

Two algorithms are available for contention resolution among the intended transmission nodes during period $T_{1}$; one is backoff based algorithm and another is $p$-persistent algorithm. The contention window size $(\mathrm{CW})$ for backoff algorithm or medium access probability $p$ for $p$-persistent algorithm should be adjusted based on the number of the active users in each beam-section.

Following the procedure described above, we can allow as many beams to be utilized as possible. Assume $T_{2}$ and $T_{3}$ are fixed. The factors that affect the overall system utilization are $p$ or $\mathrm{CW}$, the length of $T_{1}$ and the time duration between the end of a current superframe and the beginning of its next superframe, which is defined as $T_{i n t}$. Note $\mathrm{CW}$ can be approximated as the inverse of $p$. We will discuss how to adjust $p, T_{1}$ and $T_{\text {int }}$ in the next section.

\section{Adjust system parameters}

Here we discuss how to adjust system parameters introduced in III-B, which include $p, T_{1}$ and $T_{\text {int }}$. Suppose the packet arrival of each user is a relatively stable random process. We follows the stochastic-approximation approach [9] to adjust system parameters.

Define $M$ as the number of beam-sections; define $k$ as the superframe index; define $\frac{1}{W^{k}}$ as adjusting step size; define $L_{a v g}^{k}$ as the queue length (averaged over each of successful users) in superframe $k$; define $L_{\phi}$ as the system-targeted queue length; define $N_{c o l}^{k}$ as the total number of collisions divided by the number of beam-sections; define $\beta, \gamma$ and $\delta$ as the adjusting parameters.
We adjust $T_{\text {int }}$ as

$$
\left\{\begin{array}{l}
T_{\text {int }}^{k+1}=\bar{T}_{\text {int }}^{k}-\frac{\beta}{W^{k}}\left(\bar{L}_{\text {avg }}^{k}-L_{\phi}\right) \\
\bar{T}_{\text {int }}^{k}=\left(1-\frac{1}{W_{1}^{k}}\right) \bar{T}_{\text {int }}^{k-1}+\frac{1}{W^{k}} T_{\text {int }}^{k} \\
\bar{L}_{\text {avg }}^{k}=\left(1-\frac{1}{W^{k}}\right) \bar{L}_{\text {avg }}^{k-1}+\frac{1}{W^{k}} L_{\text {avg }}^{k}
\end{array}\right.
$$

such that each superframe can be fully utilized and meanwhile the average queue length of each user can approach the system-targeted value $L_{\phi}$.

Similarly, $p$ and $T_{1}$ are adjusted as

$$
\left\{\begin{array}{l}
p^{k+1}=\left(1-\frac{1}{W^{k}}\right) p^{k}-\frac{\gamma\left(N_{c o l}^{k}-M+1\right)}{W^{k}} \\
T_{1}^{k+1}=\left(1-\frac{1}{W^{k}}\right) T_{1}^{k}+\frac{\delta\left(N_{c o l}^{k}-M+1\right)}{W^{k}},
\end{array}\right.
$$

whereby, $p$ can be decreased by multiple step-sizes when collision happens and increased by one step-size when there is no collision in a super-frame; $T_{1}$ can be increased by multiple step-sizes when collision happens and decreased by one stepsize when there is no collision in a super-frame. $p$ and $T_{1}$ will converge if the packet arrival random process is relatively stable.

Note bigger step size will be more responsive to change but will lead to higher fluctuation in stochastic approximation process; on the other hand, smaller step size will keep stochastic approximation more smooth but less responsive to change. To keep balance between convergence speed and convergence smoothness, we take $W^{k}$ as

$$
W^{k}= \begin{cases}k, & k<W_{\max } \\ W_{\max }, & k \geq W_{\max }\end{cases}
$$

Finally, to let stochastic approximation run more stable, we may bound some system parameter by a minimal value and/or a maximal value. For example, since we adjust $T_{\text {int }}$ such that there are $M$ active users (one in each beam section) on average in each uplink superframe, we set the minimal value for $T_{1}$ as $700 M \mu \mathrm{s}$ in our simulation. Note $700 \mu \mathrm{s}$ is equal to $T_{s u c}+5 T_{i d}$ approximately.

\section{Compatibility issue}

Since 802.11-based WLANs have been widely deployed, now the question is how a 802.11-limited node delivers data to a multi-beam access point. There is difference between 802.11 and our proposed scheme; 802.11-limited node always follows the sender-initiated medium-access role while in our scheme, uplink medium access is access-point initiated, i.e., receiver-initiated. Fortunately, a multi-beam access point can immediately identify that a node is 802.11-limited if the access point receives a RTS from the node not during the accesspoint-coordinated-period (shown in Fig. 1). Then the access point will follow the procedure of 802.11 and immediately reply a CTS. Finally, the whole process is transparent to the 802.11-limited node.

\section{Simulation Results}

We consider a WLAN in which nodes are evenly distributed in different beam-sections and all traffic goes from nodes to the access point. All RTS/CTS/DATA/ACK packets are sent in the basic rate. The simulation tool we use is ns2. System 
TABLE I

Simulation PARAmeters

\begin{tabular}{|c|c|c|c|}
\hline Parameter & Value & Parameter & Value \\
\hline \hline Basic rate & 2 Mbits/s $)$ & Packet Size $(l)$ & $1000($ bytes $)$ \\
\hline PHY header & $192(\mu \mathrm{s})$ & $T_{1}$ & $700 \mathrm{M}(\mu \mathrm{s})$ \\
\hline$\sigma$ & $20(\mu \mathrm{s})$ & $T_{2}$ & $4000(\mu \mathrm{s})$ \\
\hline SIFS & $10(\mu \mathrm{s})$ & $T_{3}$ & $258(\mu \mathrm{s})$ \\
\hline DIFS & $40(\mu \mathrm{s})$ & $L_{\phi}$ & 1 \\
\hline RTS & $168($ bits $)$ & $\beta$ & 3 \\
\hline CTS & $136($ bits $)$ & $\gamma$ & 0.1 \\
\hline RTR & $96($ bits $)$ & $\delta$ & 0.000700 \\
\hline ACK & $112($ bits $)$ & $W_{\max }$ & 200 \\
\hline \multicolumn{2}{|r}{} \\
\end{tabular}

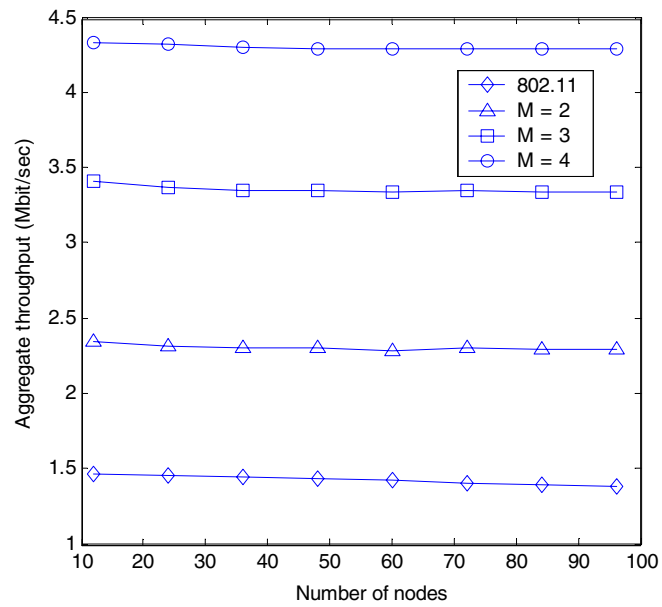

Fig. 3. Saturated throughput.

parameters are set as shown in Table I. We present two sets of simulations, one is for saturated case and another is for random-packet-arrival case.

\section{A. Saturated case}

In this set of simulation, we assume each node always has packets to transmit. We set medium access probability $p$ as $\frac{\xi}{n}$, where $\xi$ is an adjusting parameter and set as 0.5 in our study. $T_{1}$ is set as $700 M \mu \mathrm{s}$. $T_{\text {int }}$ is set to 0 . As shown in Fig. 3, our scheme can achieve 1.59, 2.33 and 2.96 times of the 802.11 throughput, when the beam number is 2,3 and 4 , respectively.

\section{B. Random packet arrival}

In this section, we assume packet arrival follows the poisson distribution. The average packet arrival rate ranges from 1 packet/user/sec to 45 packet/user/sec. The number of user is set as 24 ; the $W_{\max }$ is set as 200 . We use the stochastic approximation approach to adjust $T_{i n t}, T_{1}$ and $p$. As we desire, these system parameters can quickly converge to a certain range so that each uplink superframe can be efficiently utilized and in the meanwhile the average queue length $\bar{L}_{a v g}$ could approach the target value $T_{\phi}$, which is set as 1 in our simulation. The trace of $\bar{L}_{a v g}, T_{i n t}, T_{1}$ and $p$ is illustrated in Fig. 5 , in which average packet arrival rate is 5 packet/user/sec and the beam-section number is 3 .

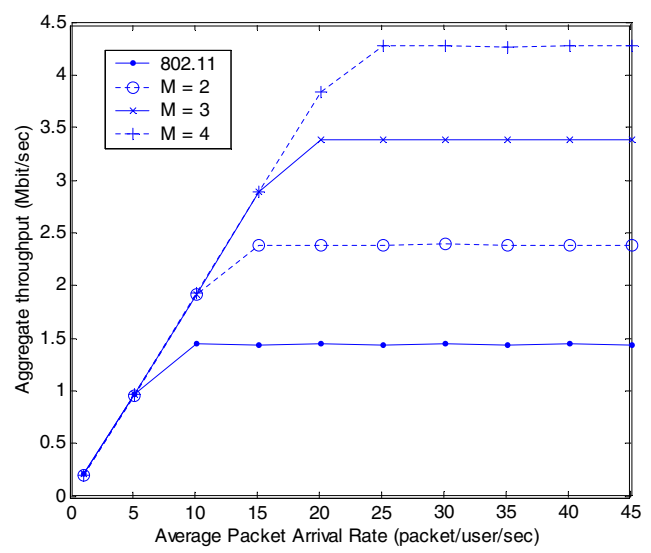

Fig. 4. Unsaturated throughput.

Finally, the simulation shows our proposed scheme significantly enlarges the zero-packet-loss throughput region by $64 \%$, $134 \%$ and $197 \%$ in comparison with 802.11 (Note buffer size in each node is 50).

\section{Conclusions}

In this paper, we propose a CSMA/CA based uplink medium access control protocol for wireless LANs with multi-beam directional-antennas access-point. Spatial reuse have been efficiently utilized by allowing as many parallel uplink data transmissions as possible. Our protocol supports backward compatibility with IEEE 802.11 (DCF mode) thus a 802.11 limited node can access the multi-beam access point transparently. Simulation results show that our scheme improves throughput significantly. Since all the nodes including the access point run in CSMA/CA based MAC, our proposed MAC protocol is not limited to the single-hop case. The enhancement of our proposed MAC protocol in multihop scenario will be our future work.

\section{REFERENCES}

[1] IEEE standard for Wireless LAN Medium Access Control (MAC) and Physical Layer (PHY) specifications, ISO/IEC 8802-11: 1999(E), Aug. 1999.

[2] A. Swaminathan, D. L. Noneaker and H. B. Russell. The receiver blocking problem in a DS mobile ad hoc networks with directional antennas. In Proc. of IEEE Military Communications Conference, Monterey CA, Oct. 2004.

[3] J. Ward and R. T. Compton Jr. High Throughput Slotted ALOHA Packet Radio Networks with Adaptive Arrays. IEEE Transactions on Communications, 41(3):460-470, March 1993.

[4] Guanghan Xu, San-Qi Li. Throughput multiplication of wireless LANs for multimedia services: SDMA protocol design. In Proc. of IEEE Global Telecommunications Conference (GLOBECOM '94), 28 Nov.2 Dec.1994, Volume: 3, Page(s): 1326 -1332.

[5] C. Sakr, T.D. Todd. Carrier-sense protocols for packet-switched smart antenna base stations. International Journal of Wireless Information Networks, 2000, Vol.7, No.3, 133-148.

[6] D. Lal, R. Toshniwal, R. Radhakrishnan, D.P. Agrawal, Jr.J. Caffery. A novel MAC layer protocol for space division multiple access in wireless ad hoc networks. In Proc.of the Eleventh International Conference on Computer Communications and Networks, 14-16 Oct. 2002, Page(s): $614-619$. 


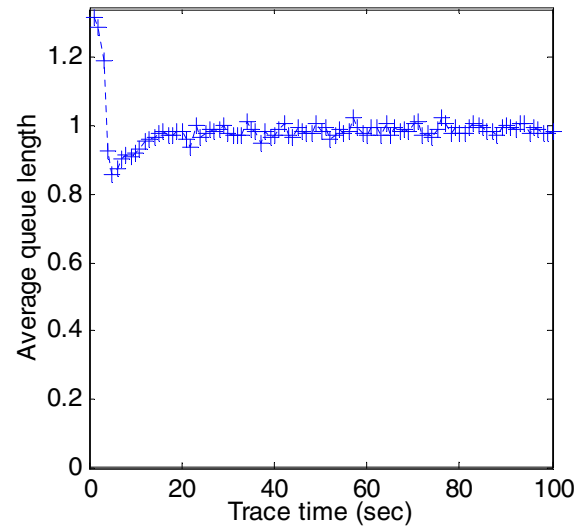

(a)

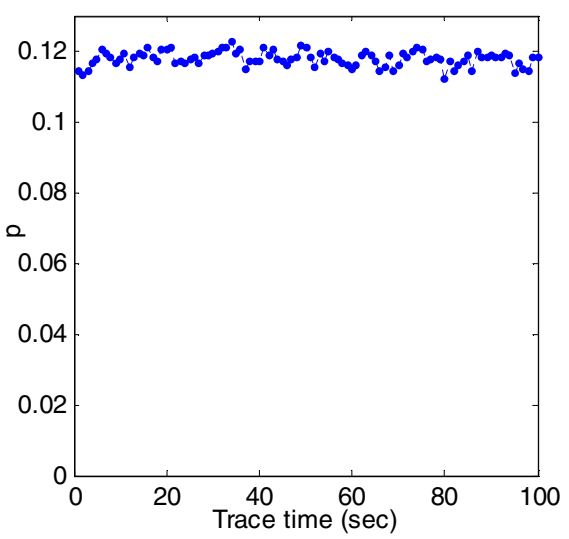

(c)

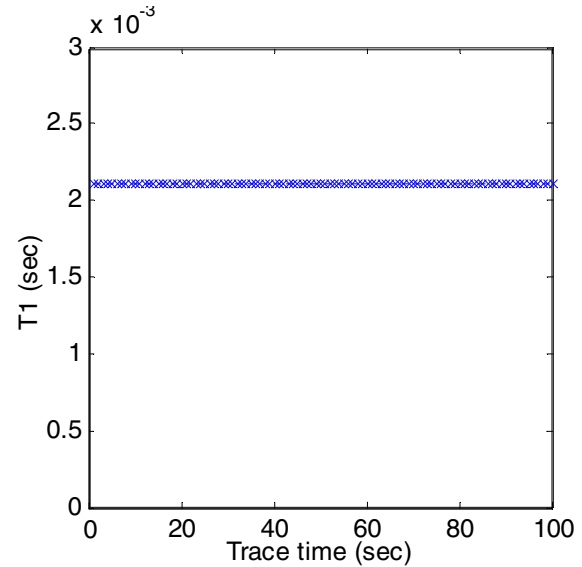

(b)

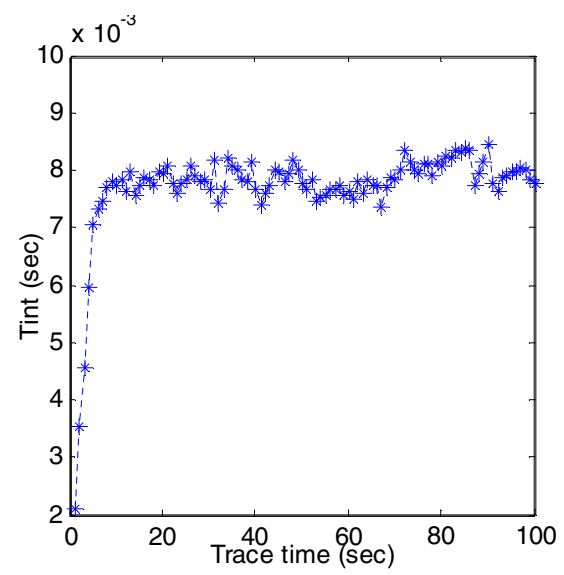

(d)

Fig. 5. Trace of system parameters: (a) $\bar{L}_{a v g}$; (b) $T_{1}$; (c) $p$; (d) $T_{\text {int }}$

[7] L. Bao and J.J. Garcia-Luna-Aceves. Transmission Scheduling in Ad Hoc Networks with Directional Antennas. In Proc. of ACM/IEEE MobiCom, Atlanta, Georgia, USA, September 23-28, 2002.

[8] T. Ren, I. Koutsopoulos, L. Tassiulas, Efficient media access protocols for wireless LANs with smart antennas. In Proc. of IEEE Wireless Communications and Networking, 16-20 March 2003, Volume: 2, Page(s) $1286-1290$.

[9] H. Kushner and G. Yin, Stochastic Approximation Algorithms and Applications. New York: Springer-Verlag, 1997. 